\title{
13 Schluss
}

Die vorliegende Studie widmete sich dem Metaphorisieren in der Interaktion audiovisueller Bilder und ihrer Zuschauer und der grundlegenden These, dass damit ein konstitutiver Zusammenhang von Wahrnehmen, Fühlen und Verstehen in den Blick kommt. Filmisches Metaphorisieren wurde als eine vom audiovisuellen Bild in spezifischer Weise modellierte und an diesem zu rekonstruierende Zuschaueraktivität entworfen: als ein über die ästhetische Organisation von Wahrnehmung modellierter Semioseprozess, als ein situativ verortetes, verkörpertes Denken und meaning-making. Von zentralem Belang hierfür sind, wie dargelegt wurde, die miteinander verwobenen Ebenen dynamischer Bildlichkeit, mit denen audiovisuelle Bilder aufgrund ihrer gestischen wie sprachlichen Ausdrucksdimension mit ihren Zuschauern kommunizieren. Damit verband sich ein Verständnis audiovisueller Bilder, das diese nicht als ein Medium der Repräsentation vorgängiger Wirklichkeiten fasst, sondern als etwas, für das der Wahrnehmungsvollzug absolut zentral ist und dem grundsätzlich eine kreative, schöpferische Dimension inhärent ist.

Um diese Idee des filmischen Metaphorisierens auszufalten und in ihre Forschungskontexte einzuordnen, habe ich zwei argumentative Bewegungen vollzogen. Diese lassen sich formelhaft als ,vom System zum Gebrauch ' und dann nochmal als, vom Sprachgebrauch zum Filme-Sehen' fassen. Damit wurde die seit Langem existierende Beziehungsgeschichte zwischen Film und Linguistik ${ }^{1}$ fortgeschrieben und ihr eine dezidiert aisthetisch-expressive, gebrauchsorientierte Perspektive hinzugefügt.

Vor dem Hintergrund einer traditionsreichen wissenschaftlichen Auseinandersetzung mit Metapher und Film, die bis zu den Anfängen der Filmtheorie zurückreicht, setzte die vorliegende Studie mit Teil I an einem ganz spezifischen Punkt an: dort, wo die einflussreiche linguistisch-philosophische Theorie konzeptueller Metaphern, mit der die Metapher nicht als kreativ-poetische oder rhetorische Figur, sondern als ein kognitives Phänomen adressiert wird, Forschungsarbeiten zur Metapher im Kontext audiovisueller Bilder motiviert. Wurde in der filmwissenschaftlichen Diskussion auch zuvor die Metapher als etwas adressiert, das kein rein sprachliches Phänomen ist und etwas mit Bedeutung/Verstehen zu tun hat, so kommen an diesem Punkt zwei weitere filmwissenschaftlich relevante Aspekte hinzu: die Frage nach verkörpertem Denken, und die nach System und Gebrauch.

So wurde zunächst die Konzeptuelle Metapherntheorie (KMT) in ihren Grundannahmen und -begrifflichkeiten erläutert, um im Anschluss daran die darauf

1 Vgl. Elsaesser/Poppe: Film.

Ә Open Access. () 2020 Christina Schmitt, publiziert von De Gruyter. (c) BY-NC-ND Dieses Werk ist lizenziert unter der Creative Commons Attribution-NonCommercial-NoDerivatives 4.0 Lizenz. https://doi.org/10.1515/9783110614619-013 
bezugnehmenden Positionen zu Metapher im Kontext audiovisueller Bilder darzulegen. Folgendes Problemfeld ging aus diesen Betrachtungen hervor: Erstens sind jene Forschungen der klassischen Denkfigur der Repräsentation verpflichtet und sehen im Gebrauch (ob nun von Sprache oder von audiovisuellen Medien) daher im Wesentlichen nur ein Moment, mit dem ein System instanziiert wird. Zweitens zeigt sich in ihnen das (zwar vielfach als obsolet erklärte, jedoch immer noch wirksame) Sender-Empfänger-Modell von Kommunikation. Drittens werden audiovisuelle Bilder als kausal-logisch zu erschließender Medientext aufgefasst. Entweder erwies sich infolgedessen das Metaphern-Verständnis von einem ,kalten‘, auf Logik abzielenden Kognitionsverständnis durchdrungen (wenn etwa kreative Metaphern als Puzzle-Rätsel aufgefasst wurden). Oder aber Verkörperung wurde auf systemische, außerfilmische Muster von Erfahrung hin entworfen (wenn die Metapher als Produkt und Automatismus betrachtet wurde). Zudem wurde jeweils insbesondere eine planerische Produzentenperspektive stark gemacht. Ein statisches Metaphernverständnis erwies sich für jene Forschungen als charakteristisch.

Vom audiovisuellen Bild als einer konkreten Form von (Wahrnehmungs-) Erfahrung hingegen wurde nicht ausgegangen: Das in besonderer Weise Sinnlich-Bildliche von Filmen, das sich in der Zeit entfaltet, sowie die körperliche Wahrnehmung dessen in einem Zuschauer-Sehen und -Hören zeigten sich in diesen auf die KMT Bezug nehmenden Ansätzen als eine Leerstelle. Die Wortsprachlichkeit, die eine dem audiovisuellen Bild integrale Ausdrucksdimension ist, fand teils gar keinen Niederschlag mehr in den Überlegungen. Und ein Verständnis von Kreativität wurde nur auf einer systemischen Ebene verortet.

Vor diesem Hintergrund wurde mit Teil II und dem dort entwickelten Verständnis filmischen Metaphorisierens durch einen grundlegenden Perspektivwechsel ein Gegenentwurf hierzu formuliert. Die durch die Konzeptuelle Metapherntheorie aufgeworfenen Fragen nach verkörpertem Denken und System/ Gebrauch im Zusammenhang mit der Metapher als einem Phänomen, das nicht rein sprachlich ist und etwas mit Verstehen und Bedeutung zu tun hat, wurden für den Kontext audiovisueller Bilder damit weiter verfolgt - allerdings ohne in der KMT selbst noch den theoretischen Ausgangspunkt zu haben. Stattdessen wurden am Gebrauch ansetzende linguistische Forschungen, die ein dynamisches Metaphernverständnis entwickelt haben, in ein produktives Resonanzverhältnis gebracht mit (zunächst einmal nicht metapherntheoretisch orientierten) Forschungen zu Ausdruck, Wahrnehmung und Verkörperung aus Medienphilosophie, Phänomenologie, Entwicklungspsychologie, philosophischer Anthropologie und Filmwissenschaft. Dabei begründete sich die metapherntheoretische Anschlussfähigkeit dieser linguistischen Forschungen zur Alltagskommunikation für die film- und medienwissenschaftlich Auseinandersetzung mit der cinematic communication in der von beiden geteilten Zeitlichkeit und Multimodalität. 
In Betrachtung der linguistischen Arbeiten zu verbalen bzw. verbal-gestischen Gesprächen wurde mit dem ,Metaphorisieren“ zunächst ein im Gebrauch verankertes Metaphernverständnis entwickelt. Vom ,Metaphorisieren` zu sprechen, unterstreicht, dass aus einer Gebrauchsperspektive ,Metapher' nichts Objekt- oder Produkthaftes ist, das benutzt werden kann. Vielmehr hat man es hierbei grundsätzlich mit einer emergenten, ein Diskursereignis gestaltenden, auf Wahrnehmungsvollzügen beruhenden Aktivität, folglich mit dynamischen Prozessen zu tun: mit Prozessen des Sehens, Fühlens und Verstehens eines Erfahrungsbereichs durch einen anderen, die an den Dynamiken der sprachlich-gestischen Artikulationen analytisch zu beschreiben sind.

In Auseinandersetzung mit den oben angeführten medien- und ausdruckstheoretischen Forschungen wiederum wurde dann ein Verständnis filmischer Kommunikation entwickelt: inwiefern ,Film‘ als ein verkörpertes, interaffektives, dynamisches Wahrnehmungsphänomen zu beschreiben ist, in das der Zuschauer mit seinem Sehen und Hören immer schon involviert zu denken ist; inwiefern von ,Bedeutung' hierbei als etwas zu sprechen ist, dass in der Zeit, in und mit der dynamischen Materialität des Medienumgangs zur Entstehung kommt - und inwiefern der Zugang hierzu in der filmischen Expressivität, die als gestische Dimension audiovisueller Bilder gefasst wurde, zu suchen ist.

Im Verständnis filmischen Metaphorisierens wurden dann die im Sprachgebrauch verankerten Metaphernforschungen mit den Forschungen zu Ausdruck, Wahrnehmung und Verkörperung miteinander verschränkt. Die klassische Denkfigur von System und Gebrauch wurde in diesem Zuge repräsentationskritisch transformiert. Von einem System ist nicht mehr als ein logisch-genealogisches Primat, als einer sich im Gebrauch manifestierenden Tiefenstruktur die Rede. Vielmehr wird noch das Diskursereignis eines jeweiligen Filme-Sehens selbst in der je spezifischen Materialität als emergentes dynamisches System aufgefasst, mit dem sich ein je eigenes, filmimmanent wirksames ,Zeichenregime‘ entfaltet.

Von Kreativität war bei den theoretischen Darlegungen zum filmischen Metaphorisieren daher immer in einer doppelten, noch ineinander verschränkten Weise die Rede - wie dies auch mit den beiden der Studie vorangestellten Motti aufgezeigt wurde. Zum einen wurde Kreativität auf die konkrete audiovisuelle Artikulation in der cinematic communication bezogen gedacht: wie in jeweils spezifischer Weise „in Bildern und Tönen ein Denken“ entworfen wird, das „eine Welt [konstitutiert]“, ${ }^{2}$ wie vom audiovisuellen Bild in seiner gestischen und wortsprachlichen Dimension das Diskursereignis des Filme-Sehens ausgestaltet ist.

2 Oliver Fahle: Zeitspaltungen. Gedächtnis und Erinnerung bei Gilles Deleuze. In: montage AV 11.1 (2002), S. 97-112, hier S. 97. 
Und zum anderen - und konstitutiv damit verschränkt - wurde dies bezogen auf die Zuschaueraktivität, auf den Rezeptionsprozess des Zuschauers selbst, der sich mit seinem Sehen und Hören in und auf ein solches Denken einlässt und es in seinem Wahrnehmen, Fühlen und Verstehen zu einer filmischen Wirklichkeit macht. $^{3}$

Exemplarisch wurden zudem Analysen zum filmischen Metaphorisieren in verschiedenen Filmformen durchgeführt (Kapitel 1, Teil II und Teil III). Primäres Anliegen war, die theoretischen Ausführungen vertiefend $\mathrm{zu}$ fundieren und zudem die breitere medienwissenschaftliche Relevanz und Tragfähigkeit des Ansatzes aufzuzeigen. Die Untersuchung filmischen Metaphorisierens erwies sich als ein flexibler deskriptiver Zugang zu audiovisuellen Bildern ganz unterschiedlicher zeitlicher Ausdehnung, Ausformung und Komplexität. So wurden mit Alfred Hitchcocks verTigo ein klassischer Hollywoodfilm und mit Tom Tykwers DER KRIEGER UND DIE KAISERIN ein zeitgenössischer deutscher Spielfilm betrachtet. Ebenso wurden ein Bankenkrisen-Beitrag aus einem ARD-Politmagazin aus dem Jahr 2008 sowie zwei Werbespots aus dem vergangenen Jahrzehnt für die Baumarktkette Hornbach und den Automobilhersteller Mercedes-Benz untersucht. Auf makro- wie mikroanalytischer Ebene wurden hierzu jeweils Ausgestaltungen von Prozessen des Metaphorisierens rekonstruierend beschrieben und auch grafisch dargelegt. Und eben in solchen Prozessbetrachtungen (und weniger in letztlichen Metaphernformulierungen) wurde das Aufschlussreiche hinsichtlich eines verkörperten Denkens gesehen, wie es sich in Diskursereignissen des FilmeSehens vollzieht.

Zwei vergleichende Beobachtungen zu den durchgeführten Analysen seien an dieser Stelle noch herausgegriffen, die zugleich zwei mögliche Stoßrichtungen für weitere Studien aufzeigen, für die die vorliegenden Untersuchungen eine Grundlage sein können.

Die eine Beobachtung betrifft die Wortsprache, die als integraler Bestandteil aller audiovisuellen Bilder erachtet werden kann, jedoch in der Regel in einem Politmagazin-Beitrag (der darin einem Nachrichtenbeitrag vergleichbar ist) eine signifikant höhere Präsenz hat als etwa in Werbe- oder Spielfilmen, wo dies sehr stark variiert - weshalb das Verhältnis von wortsprachlichem und audiovisuellem Ausdruck, ihre Relationen und Interaktionen im Metaphorisieren einen eigenen

3 Vom filmischen Metaphorisieren kann selbst noch als künstlerischer Produktion gesprochen werden, im Sinne von Michel de Certeau: Kunst des Handelns [1980]. Berlin: Merve 1988. Dies heben Kappelhoff und Greifenstein in ihren Ausführungen zur Cinematic Metaphor hervor: „,...] we understand the reception of film images as poiesis, as an act of artistic production, which is to be found in media consumption itself.“ (Kappelhoff/Greifenstein: Audiovisual Metaphors, S. 184) Vgl. ausführlich zur Poiesis des Filme-Sehens Kappelhoff: Kognition und Reflexion. 
Untersuchungsschwerpunkt darstellt. Mit Blick auf die hier durchgeführten Analysen zeigte sich etwa, dass insbesondere in den kurzen Formaten (Werbefilm, Politmagazinbeitrag) eine starke metaphorische Verdichtung darüber erfolgte, dass Redewendungen und ihre Wahrnehmungsszenarien durch die Audiovision aktiviert wurden, indem sie konkret zur Anschauung kommen (ob nun in kongruenter oder kontrastiver Weise). Dahingegen traten sinnliche Szenarien, die sprachlich artikuliert wurden, im Langformat Spielfilm in ausgedehnter Weise und auch unabhängig von einer unmittelbaren Einbindung in Redewendungen in die Ausbildung metaphorisch organisierter Bedeutungskonstitution ein.

Die zweite Beobachtung betrifft eine rhetorische Dimension. Mit Vivian Sobchack wurde gesagt, dass das, was wir ,Film‘ nennen, eine Figuration ist: nicht also etwas Gegebenes, sondern etwas im Werden Begriffenes. ${ }^{4}$ Dem sei ein Gedanke von Gilles Deleuze an die Seite gestellt, in dem es heißt, eine Erzählhandlung sei nichts Gegebenes, sondern gründe vielmehr erst im Bild ${ }^{5}$ - dieser Aspekt konnte mit den Analysen immer wieder reflektiert werden. Und Deleuze geht nun diesbezüglich noch weiter, wenn er schreibt: „Uns [...] scheint der narrative Charakter lediglich eine Konsequenz der selbst sichtbaren Bilder und ihrer direkten Kombinationen zu sein, niemals aber etwas Gegebenes““. ${ }^{6}$ In dieser Perspektive ließe sich nun der Hypothese nachgehen, dass das Überzeugende und Interesse-Weckende, wie wir es mit einem Werbefilm verbinden, oder das Diskutierende und Argumentierende eines Politmagazins bzw. eben das Erzählende von Spielfilmen über die jeweilige ästhetische Organisation der Zuschaueraktivität des filmischen Metaphorisierens zu greifen ist. Denn makroperspektivisch betrachtet stellt sich bei den untersuchten Filmformen die Modellierung dieser Aktivität jeweils unterschiedlich dar: Die dynamische Form des Metaphorisierens bei den Werbefilmen konnte als ein Ausbreiten gefasst werden, dem ein Zuspitzen bzw. Verdichten folgte und ein abschließendes Wenden bzw. Öffnen. Der Politmagazin-Beitrag hingegen war als ein gerahmtes Polarisieren mit einem Innehalten zu beschreiben. Für die Spielfilme wiederum ließ sich global gesehen eher von einem vielfachen Verzweigen sprechen.

Im Feld der interdisziplinären und zugleich wesentlich linguistisch geprägten Metaphernforschung, wie sie sich in den letzten Jahrzehnten durch die Impulse der Konzeptuellen Metapherntheorie herausgebildet hat, gibt es ein starkes Interesse an der Auseinandersetzung mit audiovisuellen Bildern. Vor jenem Hintergrund versteht sich die vorliegende Studie als ein Grundlagenbeitrag, um in diese

4 Vgl. Sobchack: The Address of the Eye, S. 54, 60.

5 Deleuze: Das Zeit-Bild, S. 47.

6 Ebd., S. 43. 
Forschungen eine ausdruckstheoretisch fundierte filmwissenschaftliche Expertise zu solchen Bildern einzuführen. Für die Filmwissenschaft wiederum führt die Arbeit die innerfachliche Beschäftigung mit der Metapher - als Denkfigur für die Frage nach Bedeutung und Verstehen - fort. Sie legt mit dem filmischen Metaphorisieren einen Ansatz dar, für den die filmische Expressivität zentral ist, und diese zu wortsprachlichen Artikulationen, die darin zum Ausdruck kommen, ins Verhältnis gesetzt wird. Indem linguistische Forschungen zur Metapher, zu verkörpertem Denken und meaning-making in der Face-to-Face-Kommunikation hierfür in ein produktives Verhältnis zur Forschung zur cinematic communication, zum Akt des Filme-Sehens gebracht werden, lässt sich die Studie zudem für die breitere film- und medienwissenschaftliche Debatte um Bedeutung und Verstehen audiovisueller Bilder fruchtbar machen.

Im Ganzen betrachtet zeigt die Untersuchung somit auf diese Weise die Kommunikation audiovisueller Bilder im Licht filmischen Metaphorisierens: als ein situativ verortetes, aus einer vielschichtigen Ausdruckshaftigkeit hervorgehendes verkörpertes Denken und meaning-making. Wo Verstehen und Bedeutung kreative Prozesse sind: etwas Fluides, das in der Zeit des Filme-Sehens, in und mit der dynamischen Materialität des Medienumgangs, im Wahrnehmen und Fühlen, in der vom audiovisuellen Bild modellierten Zuschaueraktivität zur Existenz kommt. 
